\title{
Planetary orbits in the elliptic restricted problem. V. The ADS 11060 system
}

\author{
D. Benest ${ }^{\star}$ \\ CNRS UMR 6529 "Cassini” \\ OCA Observatoire de Nice, BP 4229, 06304 Nice Cedex 4, France
}

Received 20 December 2000 / Accepted 19 December 2002

\begin{abstract}
Numerical simulations are made within the framework of the elliptic plane restricted three-body problem, in order to determine whether stable orbits exist for planets around one of the two components in double stars. The ADS 11060 system is investigated here. Large stable planetary orbits, already known to exist (more precisely, known to be possible from the dynamical point of view) through a systematic exploration of the circular model and for several cases of the elliptic model (Sun-Jupiter, $\alpha$ Centauri, Sirius, $\eta$ Coronae Borealis and ADS 12033), are found to exist around ADS 11060 A and ADS 11060 B up to distances from each star of the order of more than half the binary's periastron separation. But, in this case, nearly circular stable planetary orbits are not found to exist either in the so-called "habitable zone" around each star or anywhere else; this is very likely due to the high value of the eccentricity of the binary's orbit.
\end{abstract}

Key words. celestial mechanics - stars: binaries: general - stars: individual: ADS 11060 - stars: planetary systems

\section{Introduction}

The existence of planets in double star systems is nowadays beyond doubt, due to indirect detections since 1996 of exo-planets around one component of a binary (e.g., around 16 Cyg B, 55 Cnc A, $\tau$ Boo A, Gl 86 A or B) and to observations of probable proto-planetary disks around one or both components (e.g., BD+31 ${ }^{\circ} 643$, L1551, 55 Cnc, HR 4796). We do not discuss here large planetary orbits around the whole binary; for this case, see e.g. Dvorak $(1984,1986)$ together with Dvorak et al. (1989), and the discovery of such a planet from gravitational microlensing (Bennett et al. 1999).

In previous papers (Benest 1988a, 1989, 1996, 1998; hereafter referred to as Papers I, II, III and IV; see also Benest 1988b, which contains a large list of references, and Benest 1993), we showed that stable planetary orbits, around either the lightest or the heaviest component of four binary systems: $\alpha$ Centauri, Sirius, $\eta$ CrB (Coronae Borealis) and ADS 12033, exist up to distances from their primary of the order of more than half the binary's periastron separation.

This work is a continuation in the elliptic case of a systematic study of the circular case in the plane Restricted ThreeBody Problem (Benest 1974, 1975, 1976), where we explore numerically the possibility of existence of large stable orbits for the infinitesimal body (hereafter called the "planet" $\mathrm{P}$ ) around one of the two massive bodies (hereafter called the "stars": the primary of $\mathrm{P}$ and its companion are denoted S and $\mathrm{C}$, whose masses are respectively $m_{\mathrm{S}}$ and $m_{\mathrm{C}}$ ). The equations of motion are written as usual (see e.g. Szebehely 1967)

^ e-mail: benest@obs-nice.fr in a rotating-pulsating dimensionless coordinate system $(X, Y$, $\mathrm{d} X / \mathrm{d} T=U, \mathrm{~d} Y / \mathrm{d} T=V$, where $T$ is the true anomaly of $\mathrm{S}$ on its orbit; equations are given in Benest 1978) with origin in $S$, the companion $C$ lying on the $X$ axis on abscissa -1 , and are integrated using a fourth-order Runge-Kutta scheme with automatically variable step size. As noted in previous papers, the normalized mass of $\mathrm{S}$ is $\mu=m_{\mathrm{S}} /\left(m_{\mathrm{S}}+m_{\mathrm{C}}\right), e$ is the orbital eccentricity of the binary, $T_{\mathrm{o}}$ is the initial true anomaly of $\mathrm{S}$ (we have shown previously, see e.g. Paper I and Benest 1971, that we may consider only the case $T_{\mathrm{o}}=0$ ), and the initial conditions for $\mathrm{P}$ may be limited (Benest 1978) to $X_{\mathrm{o}}>0, Y_{\mathrm{o}}=0$ and $U_{\mathrm{o}}=0$; an orbit can thus be represented biunivocally by a point in the plane $\left(X_{\mathrm{o}}, V_{\mathrm{o}}\right)$, where the region of stable orbits can be easily visualized for any given value of the initial parameters $\mu$ and $e$. Practically, the $\left(X_{\mathrm{o}}, V_{\mathrm{o}}\right)$ plane is explored for $0 \leq X_{\mathrm{o}} \leq 1$ and $-3 \leq V_{\mathrm{o}} \leq 3$ with a grid $\Delta X_{\mathrm{o}}=0.0125-\Delta V_{\mathrm{o}}=0.05$; establishing with a fairly good precision the limit between stable and unstable regions would then require the computation of about 10000 orbits for each set of initial parameters $(\mu, e)$. Note that, due to the variable step size (computed at each time step proportionally to the distance of $\mathrm{P}$ to the nearest star $\mathrm{S}$ or $\mathrm{C}$, and to the inverse of the velocity), orbits of little dimensions or having many close encounters with one star need more and more computational time. The computation has been made on Digital "Alpha" machines at the Observatory of Nice.

Our definition for stability is as follows: a planetary orbit is called stable when there has been neither collision (with a star, S or C) nor escape (i.e. the regularity of the revolution of $\mathrm{P}$ around $\mathrm{S}$ is interrupted; for more details, see e.g. Benest 1988b) during a given number $N$ of revolutions of the binary. As for the 
previous studies, we have taken $N=100$ which corresponds, for a typical binary's orbital period of 50 to 100 years, to 5000 to 10000 years; this is of course very short compared with the lifetime of stars, but experiences indicated that instabilities appear generally inside this time span: it seemed that only orbits very near the stability limit may be revealed unstable by a longer integration, and this could imply only a little shift of the limit (see Benest 1988b); however, recent calculations (this paper and Benest \& Gonczi 1999) show that it might be indeed less simple.

A complete exploration of the plane elliptic problem would require to consider a great number of sets $(\mu, e)$, each parameter ranging independently from 0 to 1 . In a first stage, it seemed more reasonable to take values of $\mu$ and $e$ corresponding to real double stars; for every such system, we make two studies: one around the heaviest component $\mathrm{A}$ (parameter set $\mu_{\mathrm{A}}, e$ ) and one around the lightest component $\mathrm{B}$ (parameter set $\mu_{\mathrm{B}}, e$ ). In Paper I, we began the study with our nearest neighbour: the $\alpha$ Centauri system $\left(\mu_{\mathrm{A}}=0.55, \mu_{\mathrm{B}}=0.45, e=0.52\right)-$ note that other and longer simulations about $\alpha$ Centauri have recently globally confirmed our results (Wiegert \& Holman 1997; see also Holman \& Wiegert 1999) -; in Paper II, we continued with the brightest star in the Earth' sky and one of the most famous and amazing binaries: Sirius $\left(\mu_{\mathrm{A}}=2 / 3, \mu_{\mathrm{B}}=1 / 3, e=0.592\right)$; in Papers III and IV, we investigated two systems which mass ratio is the same as for $\alpha$ Centauri but with respectively a lower and a higher value of the binary's orbital eccentricity: $\eta \mathrm{CrB}$ $(e=0.28)$ and $\operatorname{ADS} 12033(e=0.83)$. Here, we go on with the exploration of the ADS 11060 system, which mass ratio is also the same as for $\alpha$ Centauri, $\eta$ CrB and ADS 12033, but with a much higher value for the binary's orbital eccentricity: $e=0.96$.

\section{Planetary orbits in the ADS 11060 system}

The binary star ADS 11060 (alias HD 165590, BD+21³302, SAO 85723, HIP 88637, STT $341 \mathrm{AB}$ ) is fairly near: around 40 pc (post-Hipparcos parallax $\approx 0.0268^{\prime \prime}$; see Söderhjelm 1999). The two stars are, as in the $\alpha$ Centauri, $\eta \mathrm{CrB}$ and ADS 12033 systems, nearly of solar spectral type (i.e. not far from $\mathrm{G} 2 \mathrm{~V}$ ); and their masses are thus also similar to the Sun's mass: $m_{\mathrm{A}}$ should be a little more than $1 M_{\odot}$, and $m_{\mathrm{B}}$ a little less than $1 M_{\odot}$, so it seemed reasonable to take for the normalized masses the same values as for the three systems cited above, i.e. $\mu_{\mathrm{A}}=0.55$ and $\mu_{\mathrm{B}}=0.45$. The binary's orbital eccentricity is 0.96 (Söderhjelm 1999); it is not a close binary stricto sensu: $a \approx 0.256^{\prime \prime}$, which gives, taking into account the distance, a mean separation of $\approx 9.55 \mathrm{AU}$; but the high eccentricity leads to a periastron distance as low as $\approx 0.4 \mathrm{AU}$, which means that realistic stable planetary orbits around each component would be near their parent star; nevertheless, detection of giant exoplanets orbiting very near, e.g., 51 Peg or upsilon Andromedae shows that such a location is not unrealistic.

Figures 1 and 2 present the stable region in the $\left(X_{0}, V_{\mathrm{o}}\right)$ plane for planetary orbits around ADS 11060 B (Fig. 1) and around ADS $11060 \mathrm{~A}$ (Fig. 2) for $T_{\mathrm{o}}=0$, i.e. for the binary initially in its minimum separation (ADS $11060 \mathrm{~A}$ and B initially

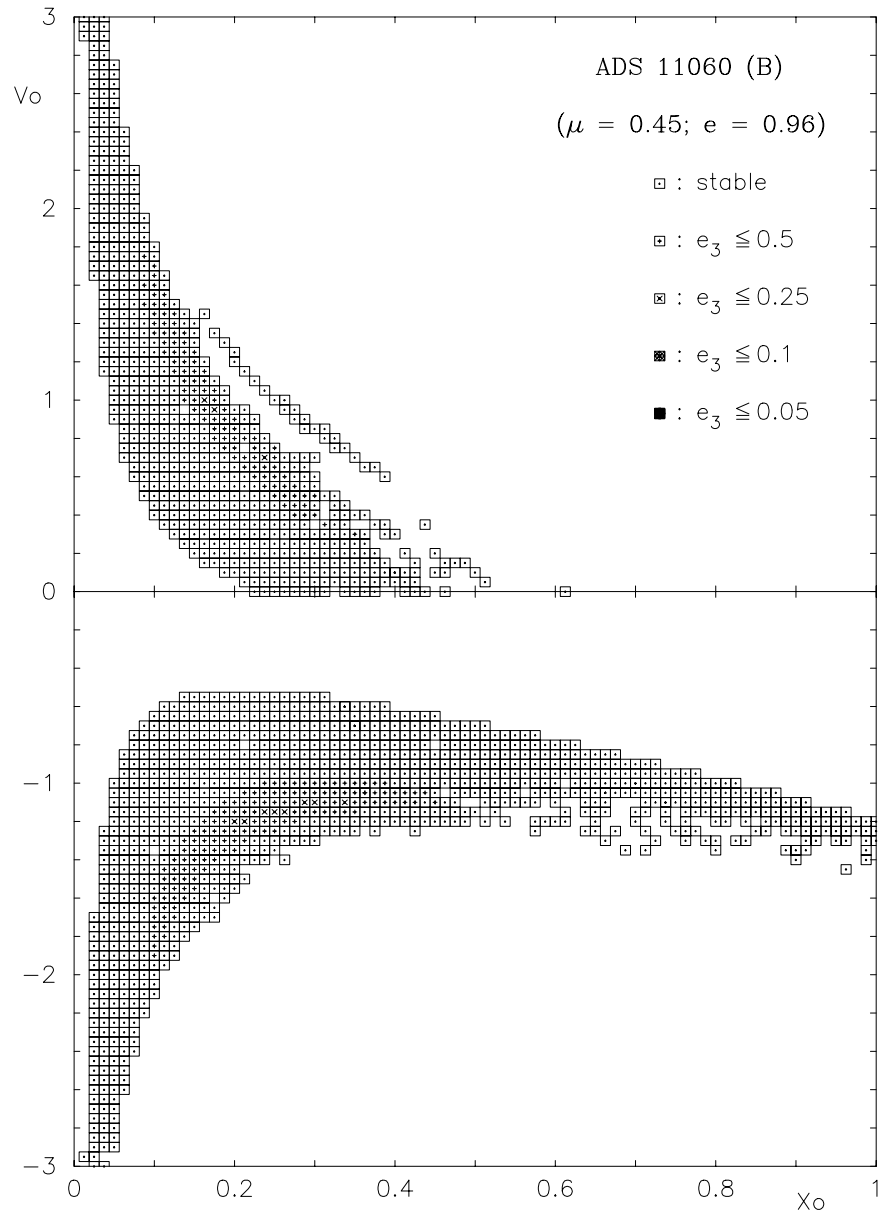

Fig. 1. Region of stability in the $\left(X_{0}, V_{\mathrm{o}}\right)$ plane (dimensionless coordinates in the rotating-pulsating frame; see text for details) for planetary orbits around ADS $11060 \mathrm{~B}$ for $T_{\mathrm{o}}=0$ (binary initially at periastron); the stable region (computation during 100 revolutions of the binary) is represented by the squares (see text for details); the pointed (resp. crossed, $\times$-ed, asterisked and filled) squares indicate planetary orbits whose eccentricity $e_{3}$ stays under the respective values given on the graph.

at their periastron. As said before, the coordinate system is dimensionless; therefore, the unit of distance $-X_{\mathrm{o}}=1 .-$ is here the value of the periastron distance of the binary, i.e. $\approx 0.4 \mathrm{AU}$ ). The presentation of the graphs is as follows: each point of the grid cited above being representative of an orbit, when this orbit is unstable, the corresponding point is not indicated on the graph; when this orbit is stable (in the sense defined above), it is surrounded with a square whose center is at the corresponding point and whose side has the same value as the step of the grid. Moreover, for a stable orbit, the drawing inside the square (point, cross, $\times$, asterisk, or totally filled square) indicates the maximum value of the eccentricity $\left(e_{3}\right)$ of the planetary orbit during the integration time. Of course, in such a graph, a square is NOT an orbit, but is here a practical way to represent an orbit and visualize the set (or the sets) of the stable orbits.

Generally speaking, the shape of the stable region is not very different from the previously studied systems (Papers I to IV): the stability zone is also separated into two regions, corresponding roughly to direct $\left(V_{\mathrm{o}}>0\right)$ and retrograde $\left(V_{\mathrm{o}}<0\right)$ 


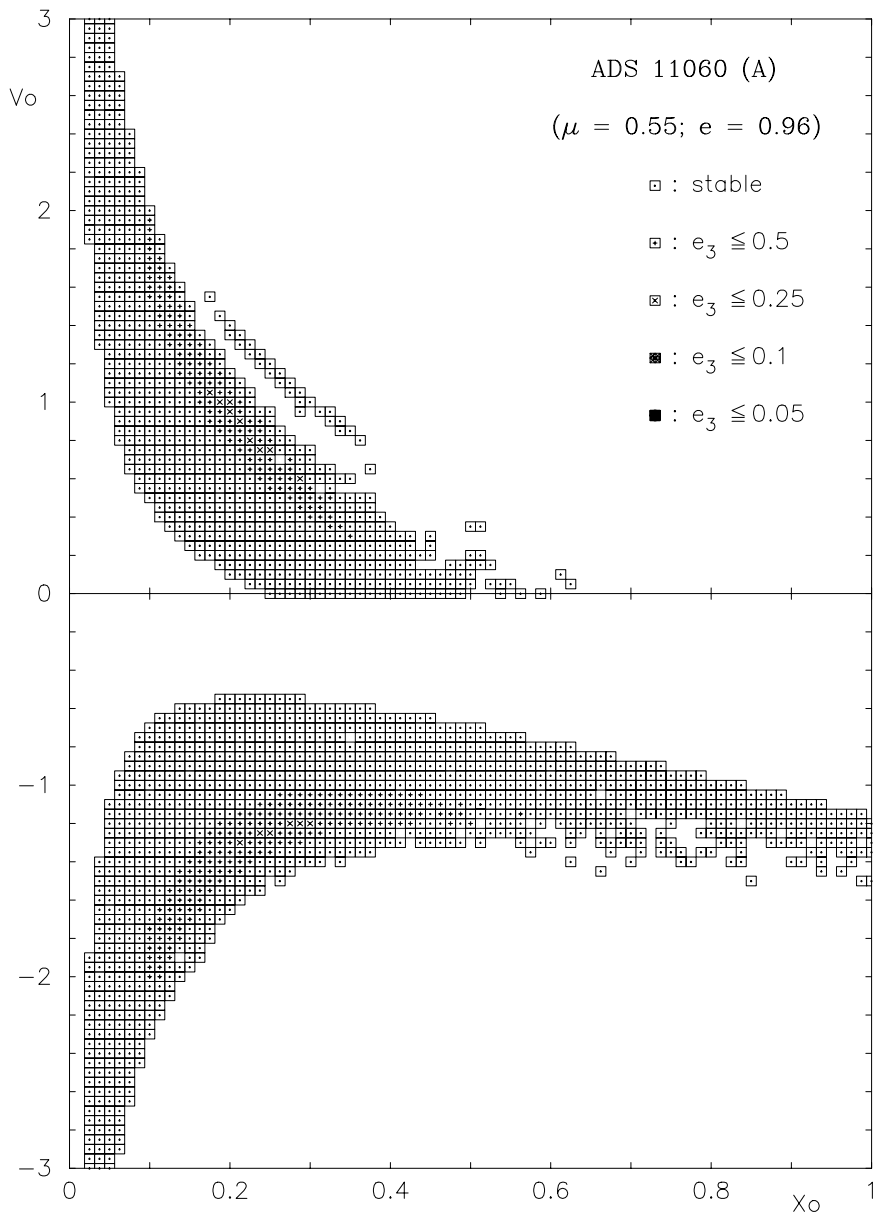

Fig. 2. Region of stability for planetary orbits around ADS 11060 A; same notations as in Fig. 1.

orbits in the rotating-pulsating frame; moreover, retrograde planetary orbits (in the rotating frame; in fixed axes, these orbits may be direct, see Benest 1978 for details) are more stable than direct ones. The stable region is surrounded by a chaotic zone (the "grey region" of Rabl \& Dvorak 1988), where appear "islands" prolonging the most advanced "cape" of stability, i.e. the largest stable planetary orbits - note that there exist also "lakes" of unstability inside the stability zone (the empty squares). In all cases (including the present paper), the most remote stable orbits (as said before, they are the retrograde ones in the rotating frame) are farther around A, the heaviest component of the binary, than around $\mathrm{B}$, the lightest one; this was presented in Table 1 of Paper IV, which is to be completed by the results of the present paper where the most remote stable orbits have $X_{\mathrm{o}}$ at least equal to 1 (see Figs. 1 and 2).

In Paper III, we had conjectured that the difference of eccentricity of the three first studied binaries $(e=0.592$ for Sirius, 0.52 for $\alpha$ Centauri and 0.28 for $\eta \mathrm{CrB}$ ) could play a role, but the results presented in Paper IV (for a high value of the binary's orbital eccentricity $-e=0.83$ !) seemed to show that it might not be the case: no significant difference was seen in the value of $X_{\mathrm{o}}$ for the most remote stable orbits in the three systems which have same value of $\mu$ and increasing value of $e$ ( $\alpha$ Centauri, $\eta \mathrm{CrB}$ and ADS 12033). However, results presented here indicate that for this very high value of $e(0.96)$,
Table 1. Largest stable direct (in the rotating frame) planetary orbits in the $\alpha$ Centauri, $\eta \mathrm{CrB}$, ADS 12033 and ADS 11060 systems; approximative values of $X_{\mathrm{o}}$ ("islands" are not taken into account).

\begin{tabular}{lllll}
\hline \hline$e$ & Star & $\mu$ & $X_{\mathrm{o}}$ & ref. \\
\hline 0.28 & $\eta$ CrB A & 0.55 & 0.45 & Paper III \\
& $\eta$ CrB B & 0.45 & 0.41 & \\
0.52 & $\alpha$ Cen A & 0.55 & 0.45 & Paper I \\
& $\alpha$ Cen B & 0.45 & 0.41 & \\
0.83 & ADS 12033 A & 0.55 & 0.45 & Paper IV \\
& ADS 12033 B & 0.45 & 0.41 & \\
0.96 & ADS 11060 A & 0.55 & 0.50 & this paper \\
& ADS 11060 B & 0.45 & 0.45 & \\
\hline
\end{tabular}

the extension of the stability zones (as much for the direct orbits as for the retrograde ones) is greater than for the three systems cited above: Table 1 gives the approximate value of $X_{\mathrm{o}}$ for the most remote stable direct (in the rotating frame) orbits for the four systems which have the same mass ratio $\mu$.

\subsection{Nearly circular planetary orbits}

But, besides, we may ask how many, amongst all the stable planetary orbits, are "nearly circular", i.e. do keep their eccentricity (hereafter noted $e_{3}$ ) under a low value for a long time. This is an important question from the point of view of bioastronomy: these nearly circular planetary orbits are supposed to keep more stable climatic conditions than eccentric ones; moreover, if these nearly circular orbits stay in the so-called "habitable zone" - in the sense of Hart (1979; see also Kasting et al. 1993) -, and if there are actually planets revolving in these orbits (which is another problem, the cosmogonic one: can planets form in double, or multiple, systems?), then these planets could be good candidates to be habitable.

In $\left(X_{\mathrm{o}}, V_{\mathrm{o}}\right)$ graphs - like Figs. 1 and 2 -, we check the stable planetary orbits whose eccentricity $e_{3}$ around their "sun" stays under respectively 0.05 (filled squares), 0.1 (asterisks), $0.25(\times)$ and 0.5 (little cross) during the 100 revolutions of the binary corresponding to our simulation; the first value of $e_{3}$ is greater than the orbital eccentricity of Venus and the Earth (resp. 0.007 and 0.017), and the second is greater than the orbital eccentricity of Mars (0.093), so that we keep a reasonable safety margin. The main result in Papers I, III and IV was that such nearly circular planetary orbits exist up to 0.2 to 0.25 times the perihelion separation of the binary.

But here, for ADS 11060, although nearly circular planetary orbits exist too, we have not the two lowest values of $e_{3}$, and what we do have lie only in small subsets of the stability zones. Is this due to the very high eccentricity? Many other binary systems have to be studied before forming any precise conclusions.

A remark about the initial eccentricity of the planetary orbits and their related fate will close this subsection. Figure 3 indicates, in an $\left(X_{\mathrm{o}}, V_{\mathrm{o}}\right)$ graph, this initial eccentricity: the set of the orbits which are initially elliptic (neither parabolic nor hyperbolic) is, as for the stable orbits (see Figs. 1 and 2), separated 


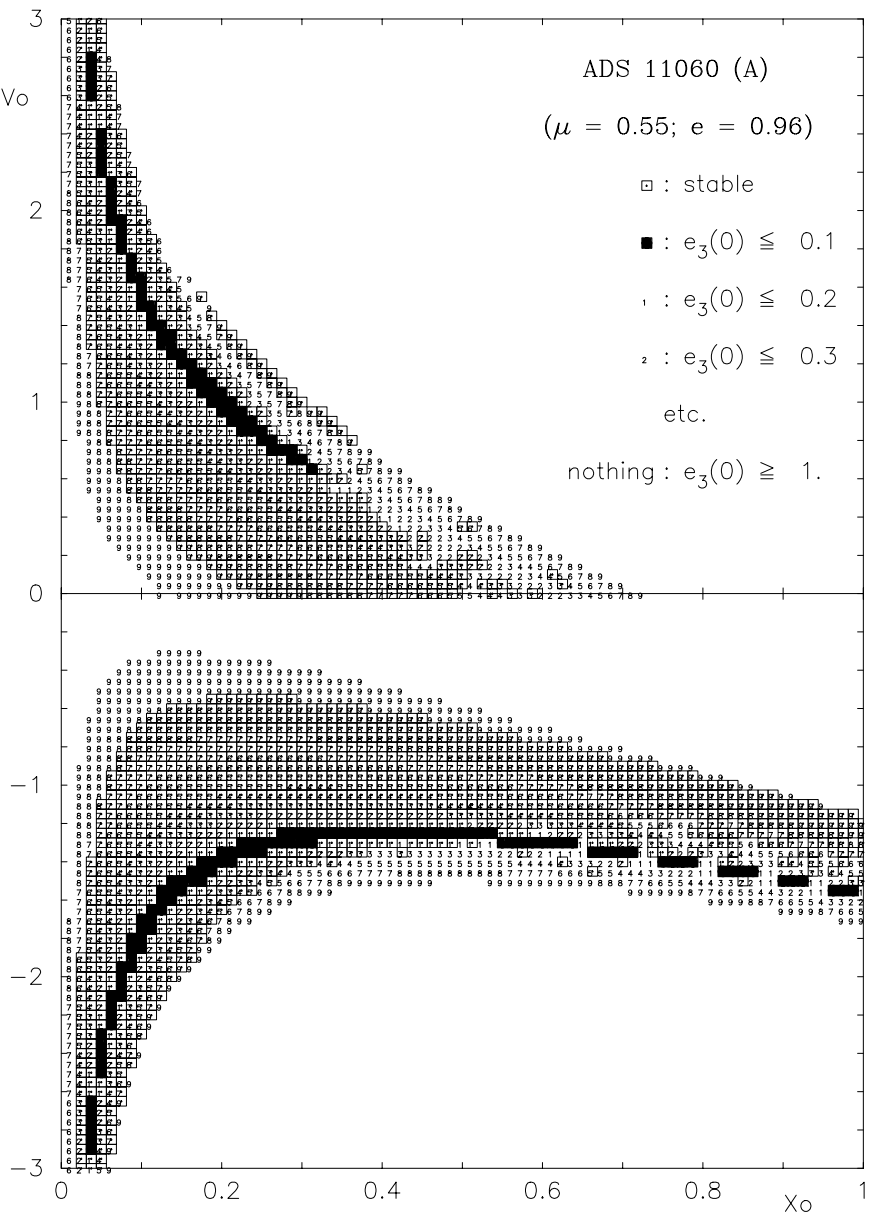

Fig. 3. Initial eccentricities for planetary orbits around ADS $11060 \mathrm{~A}$ (same coordinates as in Figs. 1 and 2; $T_{\mathrm{o}}=0$; all stable orbits are indicated by a pointed square); filled squares indicate here initiallyalmost-circular orbits (i.e. which initial eccentricity is less than 0.1); little numbers relate to the initial eccentricity when it is greater than 0.1 and less than 1 .

into two subsets, the direct and the retrograde ones (in rotatingpulsating axes). The sets of stable orbits are included inside the two subsets of initially-elliptic orbits and, moreover, generally speaking, the less the initial eccentricity, the more stable the orbit; this could be expected a priori, but it should be confirmed. Note finally that the initially almost-circular orbits $\left(e_{3}(0) \leq 0.1\right.$; filled squares $)$ shown in Fig. 3 are different from the lines of initially-circular orbits drawn in Papers I (Figs. 1 and 3) and II (Figs. 1 and 2); these latter had been extrapolated from Rabl \& Dvorak (1988) - who considered two equal stellar masses in the binary (i.e. $\mu=0.5$ ) - to our other-values-of- $\mu$ models.

\subsection{Longer computations and chaos}

As defined in the Introduction, an orbit is called stable if it stays regular during 100 revolutions of the binary; this could seem very short at first sight, but is supposed to be sufficient for a rough but extended exploration. Nevertheless, this latter faith is to be verified from time to time; this had been done for a few orbits at the border of the stability zone in the Sirius A case

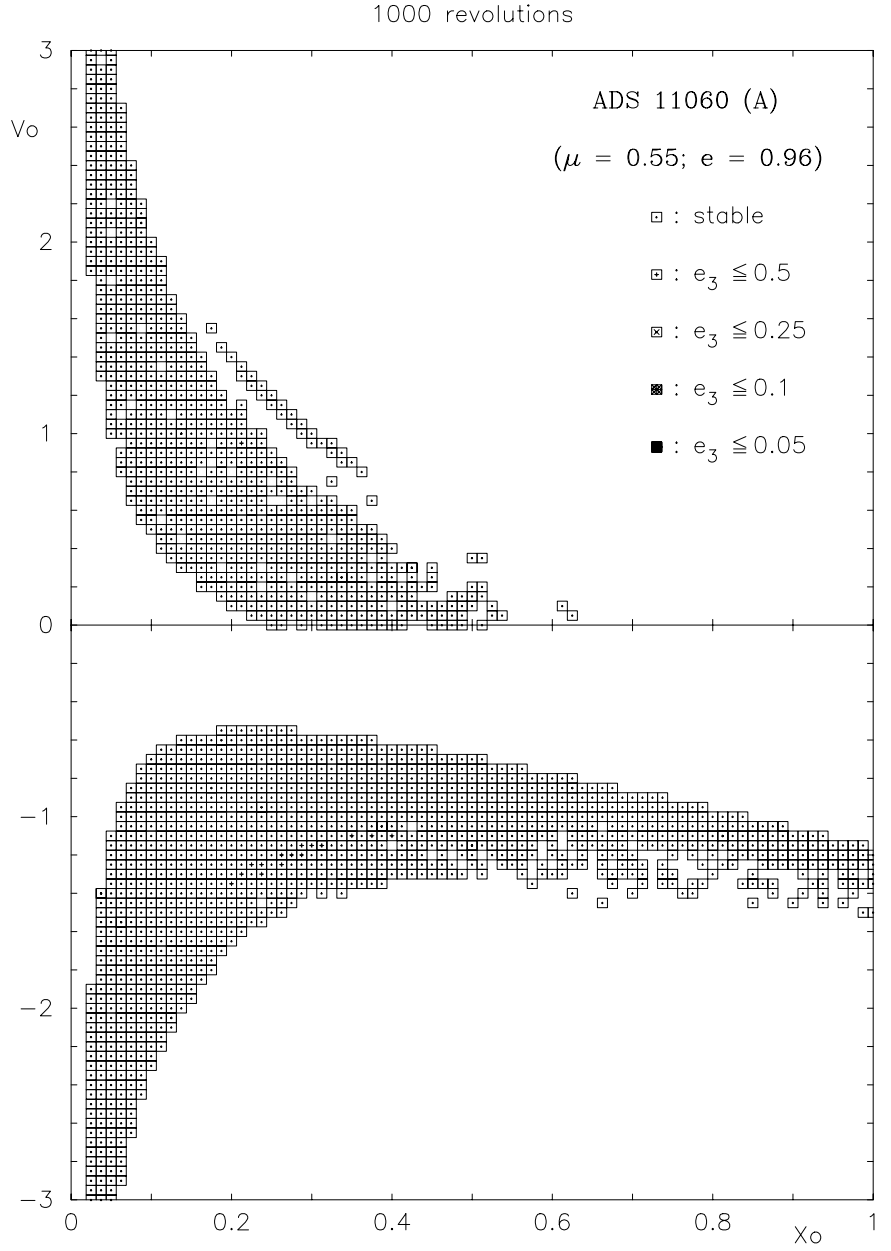

Fig. 4. Region of stability for planetary orbits around ADS 11060 A, same as Fig. 2 but for 1000 revolutions of the binary; same notations as in Fig. 1.

(Benest 1988b). Here we present a complete exploration of the $\left(X_{\mathrm{o}}, V_{\mathrm{o}}\right)$ plane for planetary orbits around ADS 11060 computed during a time span corresponding to 1000 binary revolutions, i.e. 10 times the usual simulation: Fig. 4.

At first sight, there are no important changes; the extension of the stability zone is almost the same: $X_{\mathrm{o}}$ up to 0.5 for direct orbits, and up to 1 (at least) for retrograde ones. But a more in-depth investigation reveals two main changes:

- there are many more "lakes" of instability inside the stability zones, mainly for direct orbits. Paradoxically, almost half a dozen orbits lying in "lakes" in Fig. 2 (i.e. indicating ejection or collision before the 100th revolution) are found to be stable in Fig. 4 (i.e. during 1000 revolutions). These orbits lie inside the stability zone, but not far from the border; for example, the orbit which is apparently located at $\left(X_{\mathrm{o}}=0.1375, V_{\mathrm{o}}=-1.9\right)$ has actual initial conditions of respectively $\left(X_{\mathrm{o}}=0.13749999, V_{\mathrm{o}}=-1.89999759\right)$ and $\left(X_{\mathrm{o}}=0.13749999, V_{\mathrm{o}}=-1.89999938\right)-$ the discrepancy is due to the round-offs in the computation of $X_{\mathrm{o}}$ and $V_{\mathrm{o}}$ during the complete exploration of the $\left(X_{0}, V_{\mathrm{o}}\right)$ plane-: a small difference of $2 . \times 10^{-6}$ in $V_{\mathrm{o}}$ lead to very different fates of respectively "ejection during the first binary's revolution" and 
10000 revolutions

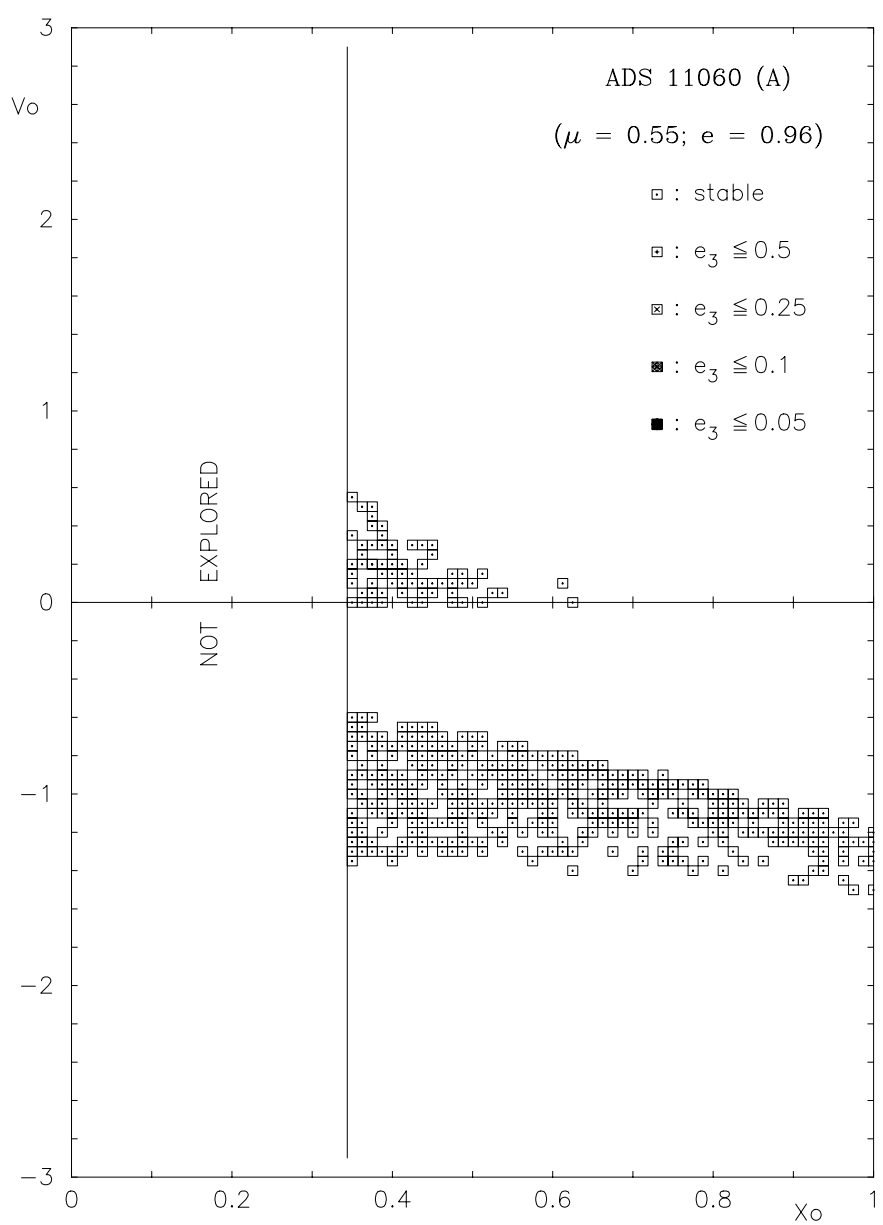

Fig. 5. Region of stability for planetary orbits around ADS 11060 A, same as Figs. 2 and 4 but for 10000 revolutions of the binary and limited to $0.35 \leq X_{\mathrm{o}} \leq 1$; same notations as in Fig. 1 .

"stability during at least 1000 revolutions"; such a behavior is one of the characteristics of chaos; therefore, a question arises: where is the actual limit between the chaotic zone and the really stable hard core (we hope it exists)? A study has been recently undertaken to obtain a quantitative measure of the stochasticity of our "stable" planetary orbits, through the computation of the Lyapunov Characteristic Indicators (see e.g. Benest \& Gonczi 1994) for some planetary orbits around $\eta \mathrm{CrB}$ A (Benest \& Gonczi 1998, 1999, 2000).

- There are fewer nearly circular stable orbits: none with $e_{3} \leq 0.25$, and the subset of $0.25<e_{3} \leq 0.5$ is much narrower and shorter, mainly for direct orbits too; moreover, an exploration, with the usual grid, of the right hand side of Fig. $4\left(0.35 \leq X_{0} \leq 1\right)$ with a longer simulation time span (10000 revolutions of the binary) does not show any significant changes, apart from a more "denticulate" shape of the "shore" of the stability zone; this seems to imply that these very wide planetary orbits, if they are not actually stable in the long term (which remains to be proven), may reveal their chaotic behavior only after this time span of 10000 revolutions; of course, we are aware that an orbit which seems stable over very long times (up to several

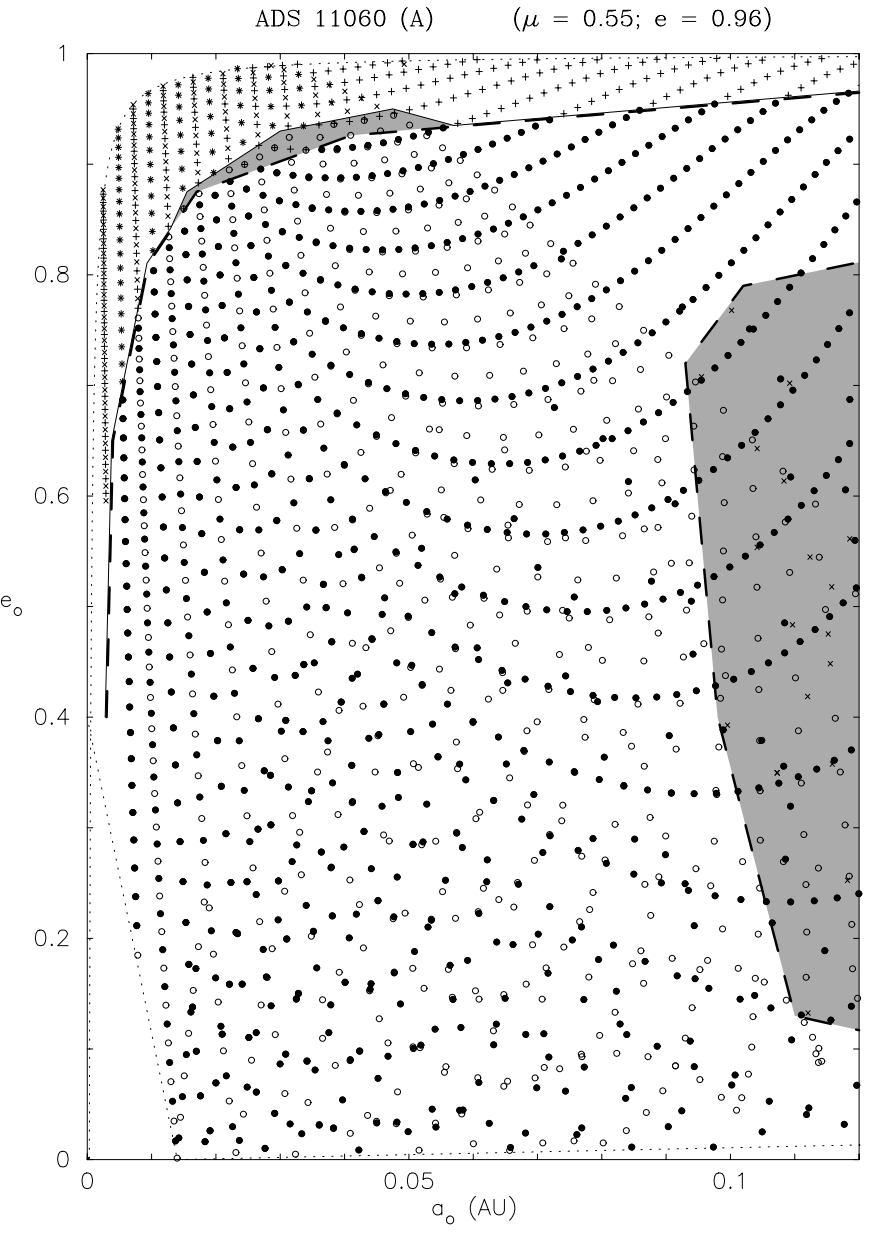

Fig. 6. Stable and unstable planetary orbits around ADS 11060 A, represented in the $\left(a_{0}, e_{0}\right)$ plane for low values of $a_{0}$. Stable orbits are represented by circles, open for direct orbits, filled for retrograde orbits; unstable orbits are represented by crosses, $\times$ for direct orbits, + for retrograde orbits. Dotted lines: boundaries for the region of orbits which lasted at least one revolution; thick dashed lines: boundaries of the region of stable orbits; thin lines: boundaries of the regions of unstable orbits; between the two latter, so-called "grey regions" may exist.

billion years) may be actually either in a "bounded" chaos state or in a so-called "stickiness" state - where the orbit escapes after a very long time, sometimes even longer than the lifetime of the Sun (see Dvorak et al. 1998 and Benest \& Gonczi 1998, 1999, 2000).

Then, to get an idea of what should happen after 1000 revolutions of the binary, we have undertaken an even longer computation: over 10000 revolutions of the primaries; not for the entire $\left(X_{0}, V_{\mathrm{o}}\right)$ plane, as this would consume very much more computing time, but only for $0.35 \leq X_{\mathrm{o}} \leq 1$ (Fig. 5). In this limited domain, we again find more "lakes" of unstability (which often link together, and sometimes even open to the vast "ocean").

\section{Another representation of the results: The $\left(a_{0}, e_{0}\right)$ plane}

This section presents the region of stability where coordinates are, instead of $\left(X_{0}, V_{\mathrm{o}}\right)$, the initial elements of the planet. As we 


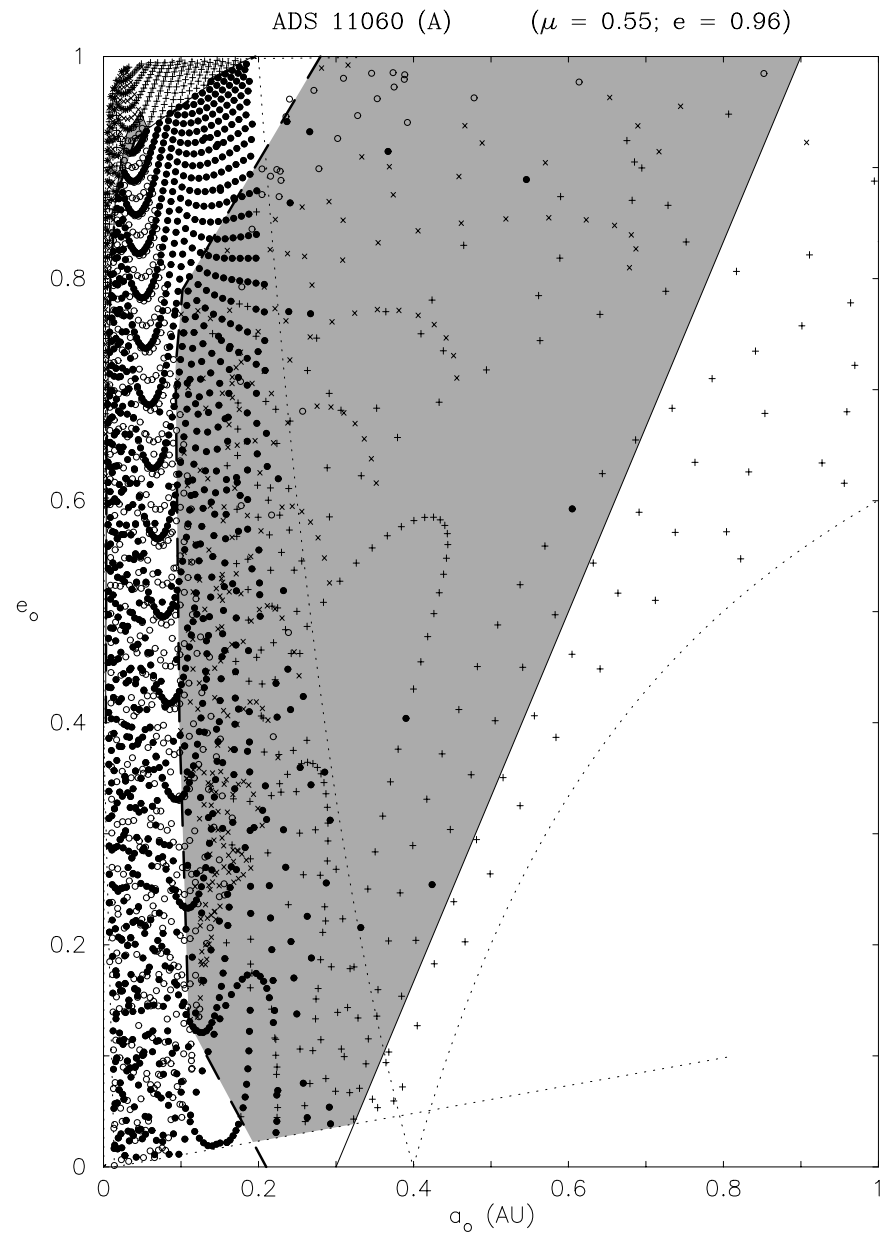

Fig. 7. Stable and unstable planetary orbits around ADS $11060 \mathrm{~A}$ in the $\left(a_{0}, e_{0}\right)$ plane up to middle values of $a_{0}$; same notations as in Fig. 6 .

are in the plane case, only the semi-major axis and the eccentricity are significant; therefore, the figures in this section are drawn in the (initial semi-major axis $a_{0}$, initial eccentricity $e_{0}$ ) plane.

In this reference frame, the set of initially elliptic planetary orbits is limited by curves showing several conditions. The first one is that the periastron distance of the planetary orbit must stay always greater than the radius $\mathcal{R}_{\mathrm{S}}$ of the parent star, otherwise a collision occurs, the computation stops and the orbit is said to be unstable; with greater reason, when the initial elements do not fulfil this first condition (i.e. when $a_{0}\left(1-e_{0}\right)$ is less than $\mathcal{R}_{\mathrm{S}}$ ), the computation either doesn't even start or, if it starts, stops very rapidly in the beginning of the first revolution of the planet; therefore, the arc of hyperbola $a_{0}=\mathcal{R}_{\mathrm{S}} /\left(1-e_{0}\right)$ (with $a_{0}>0$ and $0 \geq e_{0}<1$ ) is the left boundary of the region of orbits which last at least one revolution:

$a_{0}>\frac{\mathcal{R}_{\mathrm{S}}}{1-e_{0}}$.

The second one is that the planetary periastron must stay always under the binary's periastron distance $q$ - minus the radius $\mathcal{R}_{\mathrm{C}}$ of the companion star -, otherwise either a collision occurs or the planetary body is no longer a "satellite" of one and only one component of the binary, but its trajectory becomes another kind of orbit (see e.g. Benest 1988b); again,

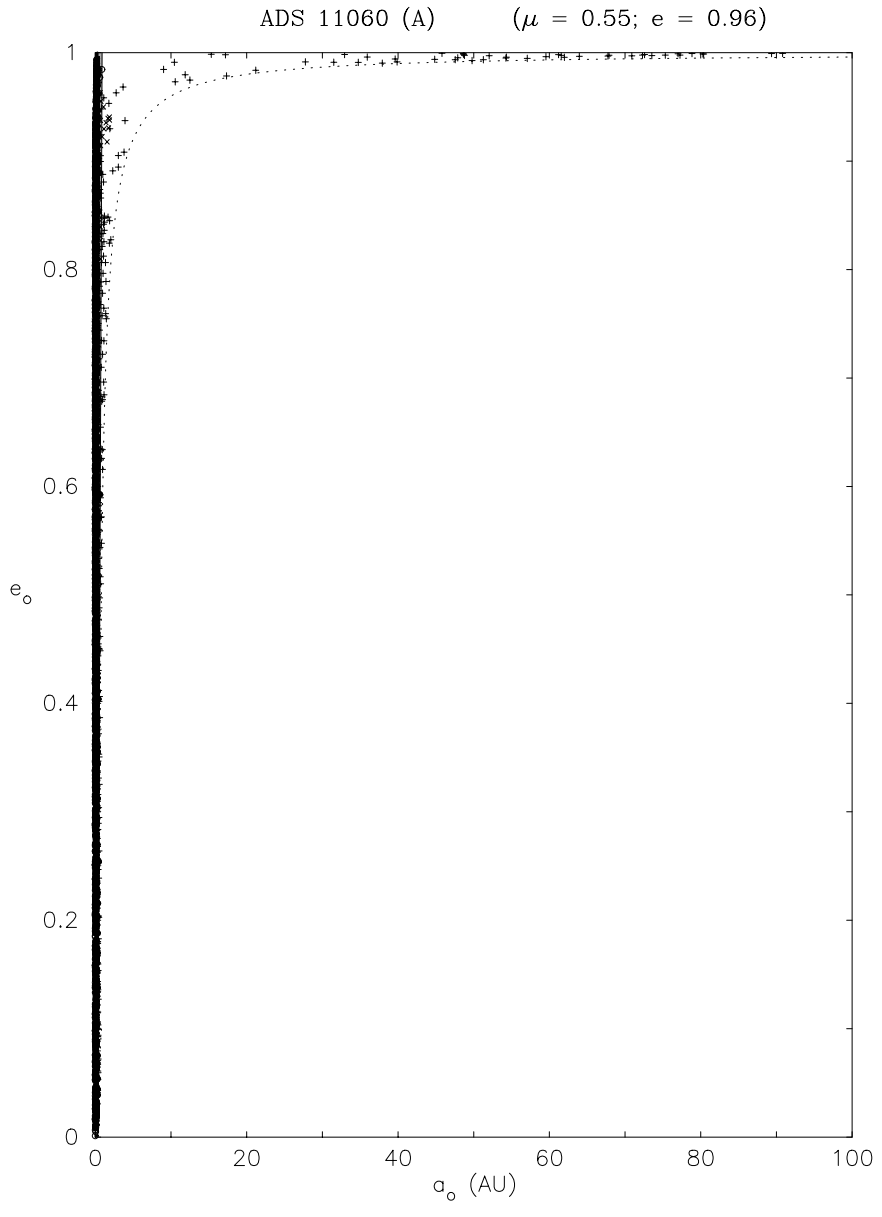

Fig. 8. Stable and unstable planetary orbits around ADS $11060 \mathrm{~A}$ in the $\left(a_{0}, e_{0}\right)$ plane up to high values of $a_{0}$; same notations as in Fig. 6.

when the initial elements do not fulfil this second condition (i.e. when $a_{0}\left(1-e_{0}\right)$ is greater than $\left.q-\mathcal{R}_{\mathrm{C}}\right)$, the computation either doesn't start or stops during the first revolution of the planet; therefore, the arc of hyperbola $a_{0}=\left(q-\mathcal{R}_{\mathrm{C}}\right) /\left(1-e_{0}\right)$ is the right boundary of the region of orbits which last at least one revolution:

$a_{0}<\frac{q-\mathcal{R}_{\mathrm{C}}}{1-e_{0}}$.

The third condition is linked to the latter, because the planetary apoastron $\left(a_{0}\left(1+e_{0}\right)\right)$ too must stay always under $q-\mathcal{R}_{\mathrm{C}}$. This could seem to be a stronger condition than the latter because $a_{0}\left(1+e_{0}\right)<a_{0}\left(1-e_{0}\right)$ but, even when the initial elements do not fulfil this third condition (i.e. when $a_{0}\left(1+e_{0}\right)$ is greater than $q-\mathcal{R}_{\mathrm{C}}$ ), the orbit may be only potentially unstable: during the first revolution of the planet, the companion star may have enough time to perturb the planetary orbit in such a way that the effective planetary apoastron become less than $q-\mathcal{R}_{\mathrm{C}}$ and the orbit may actually be stable (at least during the first revolution); therefore, the arc of hyperbola $a_{0}=\left(q-\mathcal{R}_{\mathrm{C}}\right) /\left(1+e_{0}\right)$ may be a right boundary of the region of orbits which last at least one revolution, but only in some cases, i.e. only for some values of $e_{0}$ to be determined:

$a_{0}<\frac{q-\mathcal{R}_{\mathrm{C}}}{1+e_{0}}$ 


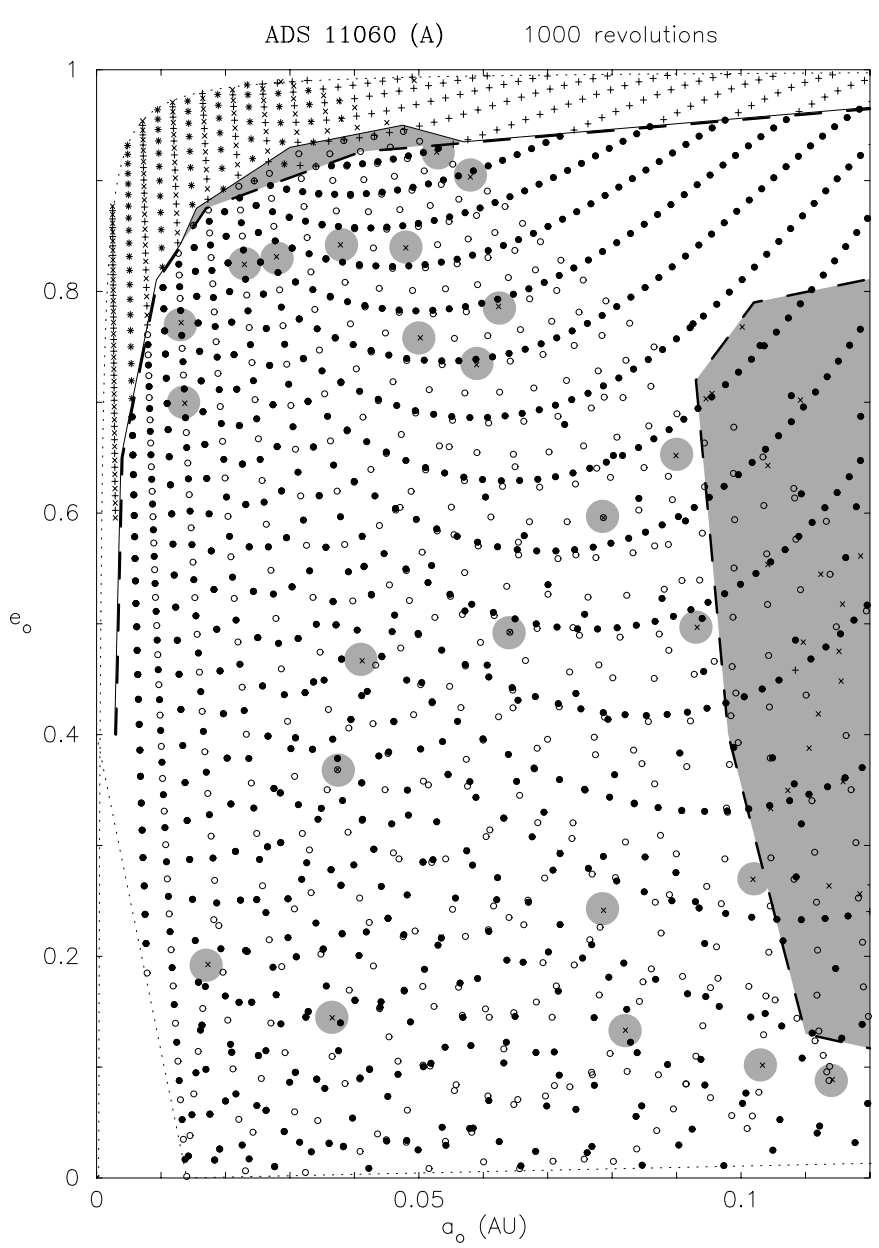

Fig. 9. Stable and unstable planetary orbits around ADS 11060 A for 1000 revolutions of the binary, represented in the $\left(a_{0}, e_{0}\right)$ plane for low values of $a_{0}$; same notations as in Fig. 6, adding small "grey regions" around unstable orbits lying inside the stable region.

These three boundaries (the two strong and the soft) are drawn as dotted lines in all the following figures.

Figures 6 to 8 represent in the $\left(a_{0}, e_{0}\right)$ plane the regions of stable and unstable (defined as above) orbits around ADS $11060 \mathrm{~A}$, scaled for three maximum values of $a_{0}: 0.12,1$ and 100. Between the hyperbolic dotted lines lies the region of orbits which lasted at least one revolution; this region seems to be limited also in the bottom left corner (low $a_{0}$, low $e_{0}$ ) where a triangle is empty, and for increasing values of $a_{0}$ and increasing values of $e_{0}$ where another triangle is empty: these two triangles are then limited also by straight dotted lines, together with the axes. Inside this delimited region, thin lines and thick dashed lines indicate boundaries of respectively stable and unstable regions; between these two boundaries lie the so-called "grey regions", as defined by Rabl \& Dvorak (1988): a large long one to the right (i.e. high $a_{0}$ and for every $e_{0}$ ) and a narrow short one to the left (i.e. low $a_{0}$ and a short interval of high values of $e_{0}$ ). Note that these inside boundaries between stable, grey and unstable regions have been determined empirically, particularly to obtain - when possible - simple convex polygonal lines; in further studies, it might be better to try to determine

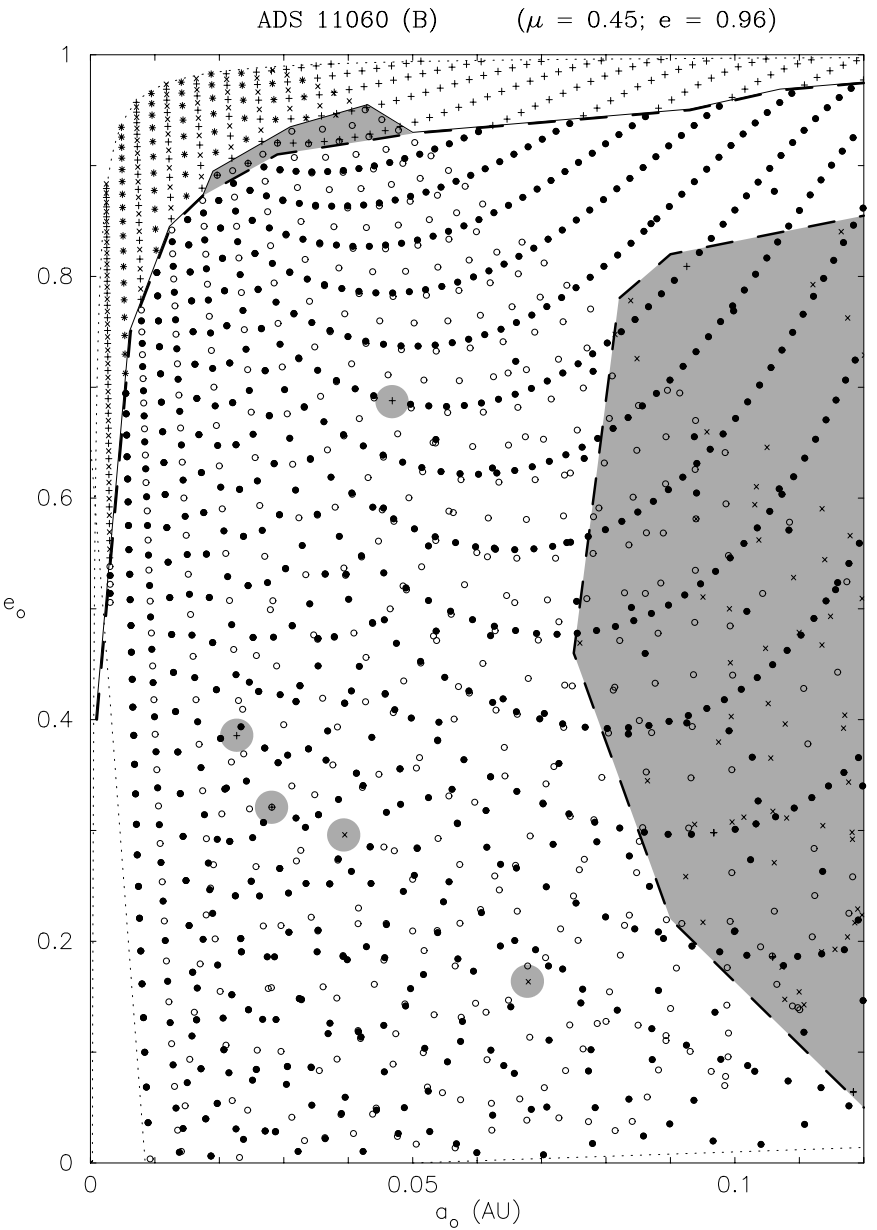

Fig. 10. Stable and unstable planetary orbits around ADS $11060 \mathrm{~B}$, represented in the $\left(a_{0}, e_{0}\right)$ plane for low values of $a_{0}$; same notations as in Figs. 6 and 9.

these boundaries through more mathematical methods such as, for example, wavelet filters.

Figure 9 represents the regions of stable and unstable orbits around ADS 11060 A for 1000 revolutions of the binary. The main change from 100 revolutions is that several isolated unstable orbits appear inside the stable region. Therefore, all boundaries are kept the same as in Fig. 6, but only adding small circular "grey regions" around these isolated unstable orbits. The change for 10000 revolutions has not been plotted because the $\left(X_{0}, V_{\mathrm{o}}\right)$ plane has not been completely explored, as explained above.

Figures 10 to 12 represent the regions of stable and unstable orbits around ADS $11060 \mathrm{~B}$. As soon as for 100 revolutions, several isolated unstable orbits already appeared inside the stable region, and are plotted as in Fig. 9 by small circular "grey regions".

Figures 7 and, to a lesser degree, 11 show that the "soft boundary" defined above $\left(a_{0}=\left(q-\mathcal{R}_{\mathrm{C}}\right) /\left(1+e_{0}\right)\right)$ is not only a theoretical one: at the right-hand side of this arc of the hyperbola and for high values of $e_{0}$, a small triangle is completely (in Fig. 7) or almost completely (Fig. 11) empty.

Such plots in the $\left(a_{0}, e_{0}\right)$ plane need to be done for other binaries to allow conclusions about the evolution of the 


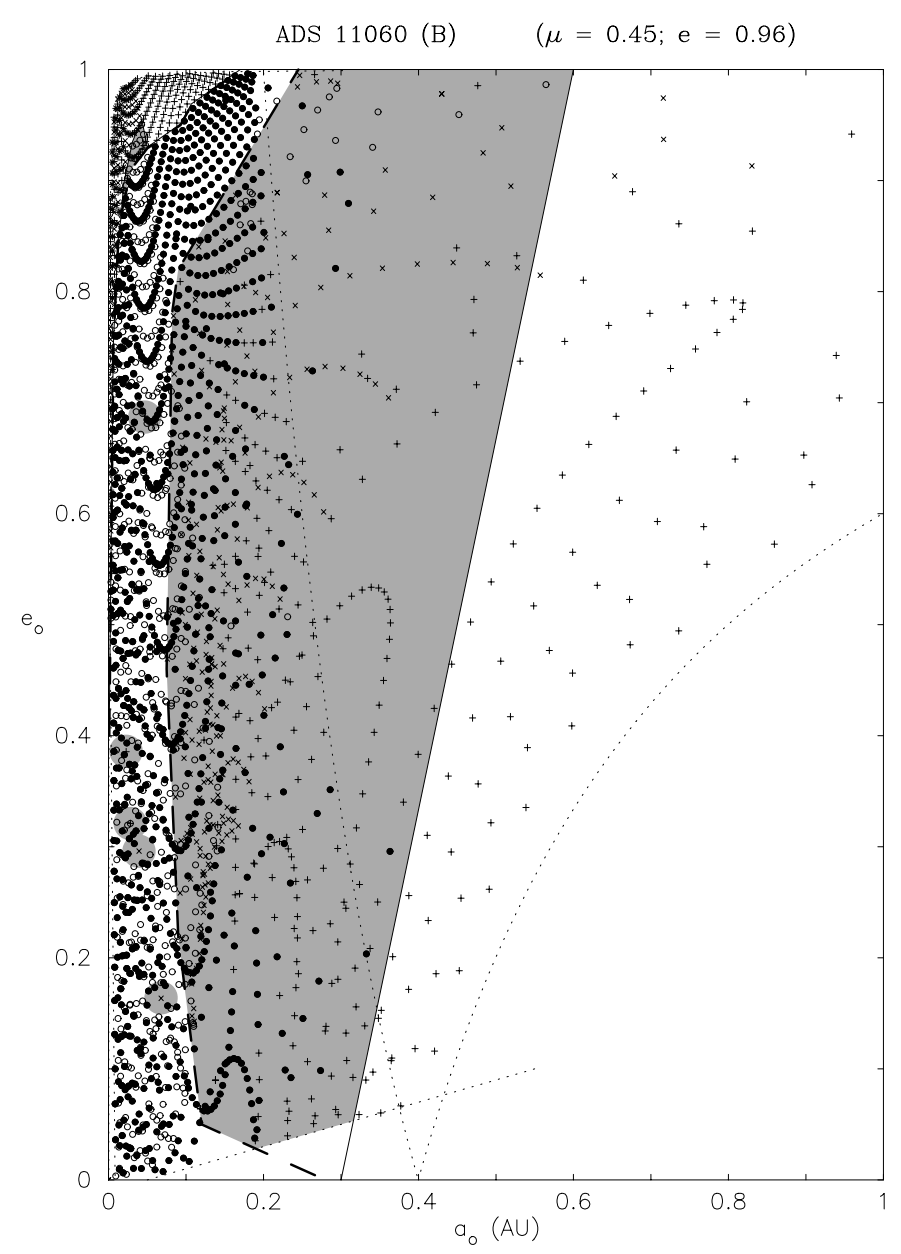

Fig. 11. Stable and unstable planetary orbits around ADS $11060 \mathrm{~B}$ in the $\left(a_{0}, e_{0}\right)$ plane up to middle values of $a_{0}$; same notations as in Figs. 6 and 9 .

boundariess between stable, grey and unstable orbits when $\mu$ and $e$ vary. Let us remark particularly that the bottom part of all the plots presented here (Figs. 6 to 12) is poorly populated; this indicates that, although orbits with initially-low-eccentricity (i.e. $\left.e_{0}<0.1\right)$ are relatively common, orbits with initially very low eccentricity (i.e. $\left.e_{0}<0.01\right)$ are much more rare for the presently studied set of initial parameters $(\mu, e)$, corresponding to the binary ADS 11060; moreover, none is initially strictly circular (i.e. $e_{0}=0$ ). But what of other values of $e$ ? A return to previously studied binaries with same value of $\mu$ ( $\alpha$ Centauri, Paper I; $\eta$ CrB, Paper III; and ADS 12033, Paper IV) is highly desirable, the goal of which would be to revisit these values, compare with ADS 11060 from this point of view, and to try to determine the actual influence of $e$; this will be undertaken in the near future.

\section{Discussion and prospects}

We limit ourselves here to the plane case (the planet's trajectory stays in the binary's orbital plane), as it has been shown that the stability of such planetary orbits is relatively insensitive to the inclination of the planet's trajectory with respect to the binary's orbital plane. Besides, recent studies about orbital coplanarity in binaries seem indicate that planetary orbital

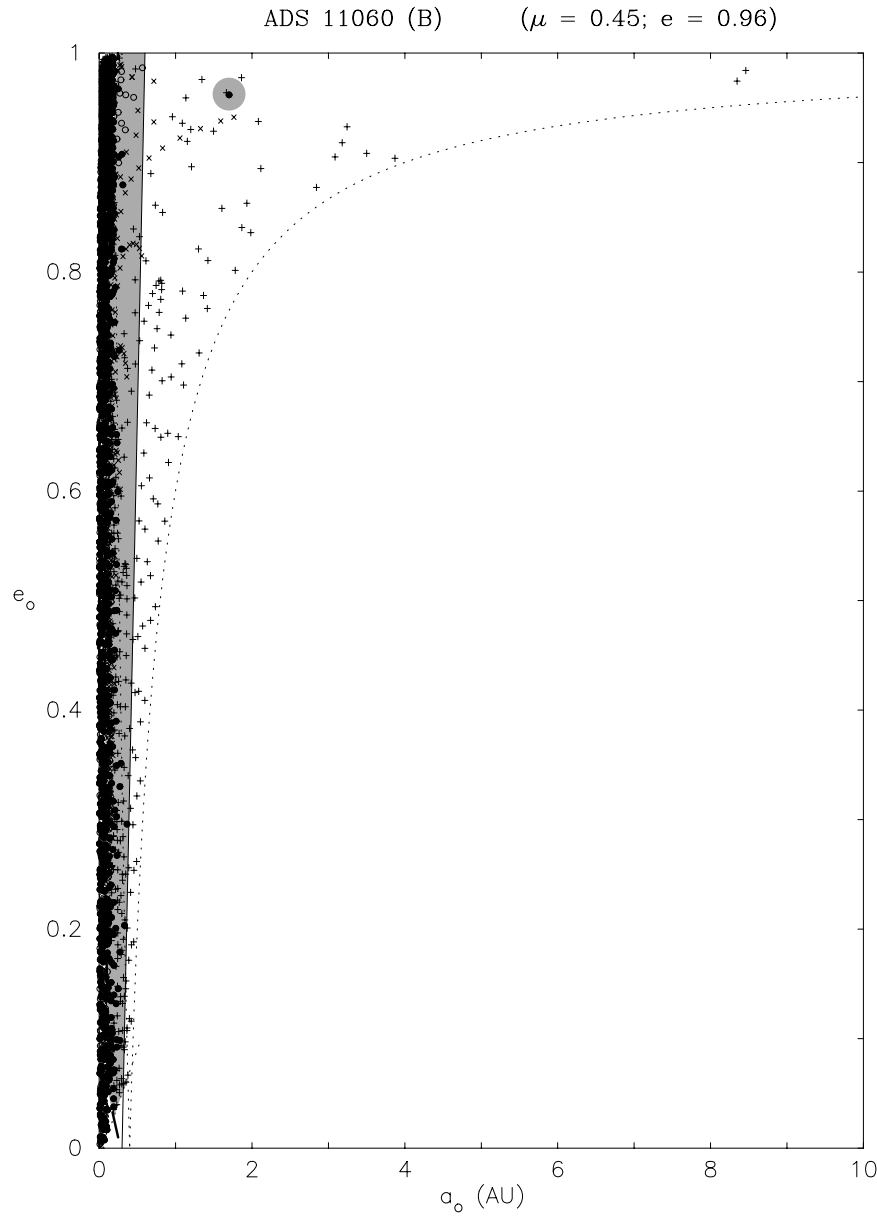

Fig. 12. Stable and unstable planetary orbits around ADS $11060 \mathrm{~B}$ in the $\left(a_{0}, e_{0}\right)$ plane up to high values of $a_{0}$; same notations as in Figs. 6 and 9 .

planes are probably not very much inclined with respect to the binary's orbital plane (see e.g. Hale 1994).

We consider here orbits for a single planet in an isolated binary. In real systems, we may expect the presence of several bodies around each star of the binary (we have several examples of multiple planetary systems, but up to now only around single stars, see e.g. Kisseleva-Eggleton et al. 2002); mutual perturbations would have therefore to be taken into account (and chaos too; see e.g. Laskar 1990). Moreover, real systems are not isolated in the Universe; for example, a very close encounter with another star or a giant molecular cloud could modify the planets' trajectories; but another kind of perturbation, more regular, can come from physically linked companion(s) of the system: in our case, ADS $11060 \mathrm{AB}$ is part of a larger system, with ADS $11060 \mathrm{C}$ - which is itself a spectroscopic binary - at $1200 \mathrm{AU}$ (Fekel et al. 1994).

Finally, this paper, together with Papers I to IV, confirms for the elliptic case the main result obtained in the circular restricted three-body problem, i.e. the existence of stable planetary orbits around one component of double stars up to a distance from their parent star of the order of, or superior to, half the periastron separation of the binary. This systematic exploration of the elliptic case has to be continued for many other nearby binaries; our final goal is to establish the limiting 
values, if they exist, of $e$ (binary's orbital eccentricity) and $\mu$ (binary's mass ratio) beyond which no such stable planetary orbits exist, together with the exploration of the limits between the hard core of billion-year-stable planetary orbits and the chaotic domain.

Acknowledgements. This research is supported by the "Programme National de Planétologie, Action Spécifique: Planètes Extrasolaires" of the "Centre National de la Recherche Scientifique". The bibliographical research has made use of the SIMBAD database, operated at CDS, Strasbourg, France.

It is a pleasure to thank Dr. R. Dvorak for gentle remarks.

\section{References}

Benest, D. 1971, A\&A, 13, 157

Benest, D. 1974, A\&A, 32, 39

Benest, D. 1975, A\&A, 45, 353

Benest, D. 1976, A\&A, 53, 231

Benest, D. 1978, Orbites de satellite dans le problème restreint, Thèse, Obs. Nice

Benest, D. 1988a, A\&A, 206, 143

Benest, D. 1988b, Celes. Mech., 43, 47

Benest, D. 1989, A\&A, 223, 361

Benest, D. 1993, Celes. Mech., 56, 45

Benest, D. 1996, A\&A, 314, 983

Benest, D. 1998, A\&A, 332, 1147
Benest, D., \& Gonczi, R. 1994, Planet. Sp. Sci., 42, 183

Benest, D., \& Gonczi, R. 1998, Earth, Moon and Planets, 81, 7

Benest, D., \& Gonczi, R. 1999, Comptes-Rendus Acad. Sci., 327 Ser. II b, 1345

Benest, D., \& Gonczi, R. 2000, in Birth and Evolution of Binary Stars, ed. B. Reinpurth, \& H. Zinnecker (Astrophysikalisches Institut Potsdam), 214

Bennett, D. P., Rhie, S. H., Becker, A. C., et al. 1999, Nature, 402, 57

Dvorak, R. 1984, Celes. Mech., 34, 369

Dvorak, R. 1986, A\&A, 167, 379

Dvorak, R., Contopoulos, G., Efthymiopoulos, C., \& Voglis, N. 1998, Planet. Sp. Sci., 46, 1567

Dvorak, R., Froeschlé, Ch., \& Froeschlé, Cl. 1989, A\&A, 226, 335

Fekel, F. C., Henry, G. W., Hampton, M. L., Fried, R., \& Morton, M. D. 1994, AJ, 108, 694

Hale, A. 1994, AJ, 107, 306

Hart, M. H. 1979, Icarus, 37, 351

Holman, M. J., \& Wiegert, P. A. 1999, AJ, 117, 621

Kasting, J. F., Whitmire, D. P., \& Reynolds, R. T. 1993, Icarus, 101, 108

Kisseleva-Eggleton, L. G., Bois, E., Rambaux, N., \& Dvorak, R. 2002, ApJ, 578, L145

Laskar, J. 1990, Icarus, 88, 266

Rabl, G., \& Dvorak, R. 1988, A\&A, 191, 385

Söderhjelm, S. 1999, A\&A, 341, 121

Szebehely, V. 1967, Theory of orbits (Acad. Press N.Y.)

Wiegert, P. A., \& Holman, M. J. 1997, AJ, 113, 1445 